\title{
Enforcing Full Arc Consistency in Asynchronous Forward Bounding Algorithm
}

\author{
Rachid Adrdor, and Lahcen Koutti
}

\begin{abstract}
The AFB_BJ ${ }^{+}$DAC $^{*}$ is the latest variant of asynchronous forward bounding algorithms used to solve Distributed Constraint Optimization Problems (DCOPs). It uses Directional Arc Consistency $\left(\mathrm{DAC}^{*}\right)$ to remove, from domains of a given DCOP, values that do not belong to its optimal solution. However, in some cases, $\mathrm{DAC}^{*}$ does not remove all suboptimal values, which causes more unnecessary research to reach the optimal solution. In this paper, to clear more and more suboptimal values from a DCOP, we use a higher level of DAC* ${ }^{*}$ called Full Directional Arc Consistency $\left(\right.$ FDAC $\left.^{*}\right)$. This level is based on reapplying $\mathrm{AC}^{*}$ several times, which gives the possibility of making more deletions and thus quickly reaching the optimal solution. Experiments on some benchmarks show that the new algorithm, AFB_BJ ${ }^{+}$FDAC ${ }^{*}$, is better in terms of communication load and computation effort.
\end{abstract}

Index Terms-DCOP, AFB_BJ ${ }^{+}$AC $^{*}$, Soft Arc Consistency, Full Directional Arc Consistency.

\section{INTRODUCTION}

$\mathbf{T}$ here are a large number of multi-agent problems that can be modeled as DCOPs such as meetings scheduling [17], sensor networks [7], [18], and so on. In a DCOP, variables, domains, and constraints are distributed among a set of agents. Each agent has full control over a subset of variables and constraints that involve them [11]. A DCOP is solved in a distributed manner via an algorithm allowing the agents to cooperate and coordinate with each other to find a solution with a minimal cost. A solution of a DCOP is a set of value assignments, each representing the value assigned to one of the variables of that DCOP. Algorithms with various search strategies have been suggested to solve DCOPs [9], [10]. Among them, there are Adopt [19], BnB-Adopt [25], BnB$\mathrm{Adopt}^{+}$[13], SyncBB [15], AFB [11], [21], AFB_BJ ${ }^{+}$[23], AFB_BJ ${ }^{+} A^{A} C^{*}[1]-[3]$, AFB_BJ ${ }^{+}{ }_{2} D A C^{*}[4]$, [5], etc.

In AFB_BJ ${ }^{+}{ }_{-}$DAC ${ }^{*}$, to find the optimal solution to a given problem, the agents synchronously exchange a current partial assignment (CPA) containing their assignments. During this process, and to reduce the number of exchanges, each agent uses directional arc consistency (DAC*) to remove any suboptimal values in its domain. The positive behavior of DAC* depends closely on DCOP to be solved in terms of its constraints and costs. This is what sometimes prevents DAC* $^{*}$ from behaving better in AFB_BJ ${ }^{+}$DAC $^{*}$ algorithm. This often occurs in DCOPs where the constraints are sparse or they are dense but most of their costs are zero. For that,

Manuscript received April 10, 2021; revised December 21, 2021. Date of publication January 18, 2022. Date of current version January 18, 2022.

Authors are with the Department of Computer Science, Faculty of Sciences,

Agadir, Morocco (e-mails: rachid.adrdor@edu.uiz.ac.ma, 1.koutti@uiz.ac.ma)

Digital Object Identifier (DOI): 10.24138/jcomss-2021-0083 we suggest in this paper to upgrade $\mathrm{DAC}^{*}$ to the next higher level, which is Full Directional Arc Consistency (FDAC*). The new algorithm is called AFB_BJ ${ }^{+}$FDAC $^{*}$ and allows agents to perform $\mathrm{AC}^{*}$ multiple times and thus remove more suboptimal values from their domains.

Our experiments on different benchmarks show the superiority of AFB_BJ ${ }^{+}{ }_{-} \mathrm{FDAC}^{*}$ algorithm in terms of communication load and computation effort.

This paper is made up of three main sections. Section II presents an overview of DCOPs, soft arc consistency rules, AFB_BJ ${ }^{+}$AC $^{*}$ algorithm, and $\mathrm{AFB} \_\mathrm{BJ}^{+}{ }^{-} \mathrm{DAC}^{*}$ algorithm. Section III gives a description of AFB_BJ ${ }^{+}$FDAC $^{*}$ algorithm. Section IV exposes the experiments carried out on some benchmarks.

\section{BACKGROUND}

\section{A. Distributed Constraint Optimization Problem (DCOP)}

A DCOP [12] is defined by 4 sets, set of agents $\mathcal{A}=$ $\left\{A_{1}, A_{2}, \ldots, A_{k}\right\}$, set of variables $\mathcal{X}=\left\{x_{1}, x_{2}, \ldots, x_{n}\right\}$, set of domains $\mathcal{D}=\left\{D_{1}, D_{2}, \ldots, D_{n}\right\}$, each $D_{i}$ is the possible values of $x_{i}$ in $\mathcal{X}$, and set of soft constraints $\mathcal{C}=\left\{C_{i j}\right.$ : $\left.D_{i} \times D_{j} \rightarrow \mathbb{R}^{+}\right\} \cup\left\{C_{i}: D_{i} \rightarrow \mathbb{R}^{+}\right\}$. In a DCOP, each agent is fully responsible for a subset of variables and the constraints that involve them.

In this paper, while maintaining the generality, we only consider DCOPs in that each agent is responsible for a single variable and that every two variables, at most, are linked by a constraint (i.e., unary or binary constraint) [20].

We consider these notations : $A_{j}$ is an agent, where $j$ is its level or rank in the default ordering. $\left(x_{j}, v_{j}\right)$ is an assignment of $A_{j}$, where $v_{j} \in D_{j}$ and $x_{j} \in \mathcal{X}$. $C_{i j}$ is a binary constraint between $x_{i}$ and $x_{j} . C_{i j}^{a c}$ is an identical copy of the $C_{i j}$ constraint, used in $\mathrm{AC}^{*}$ process. $C_{j}$ is a unary constraint on $x_{j} . C_{\phi}$ is the global zero-arity constraint that represents a lower bound of any solution of a given DCOP. $C_{\phi_{j}}$ is the local zero-arity constraint that represents the contribution value of $A_{j}$ in $C_{\phi}$ (i.e., $C_{\phi}=\sum_{A_{j} \in \mathcal{A}} C_{\phi_{j}}$ ). $U B_{j}$ is the cost of the optimal solution reached so far. $\left[A_{1}, A_{2}, \ldots, A_{n}\right]$ is the lexicographic ordering of agents (the default ordering). $\Gamma\left(x_{j}\right)=\left\{\Gamma^{-}: x_{i} \in \mathcal{X} \mid C_{i j} \in \mathcal{C}, i<j\right\} \cup\left\{\Gamma^{+}: x_{i} \in\right.$ $\left.\mathcal{X} \mid C_{i j} \in \mathcal{C}, i>j\right\}$ is the set of neighbors of $A_{j} . \Gamma^{-}$ (resp. $\Gamma^{+}$) is the set of neighbors with a higher priority (resp. with a lower priority). $Y=Y^{j}=\left[\left(x_{1}, v_{1}\right), \ldots,\left(x_{j}, v_{j}\right)\right]$ is a current partial assignment (CPA). $v_{j}^{*}$ is the optimal value of $A_{j} . l b_{k}[i]\left[v_{j}\right]\left(Y^{j}\right)$ are the lower bounds of a lower neighbor $A_{k}$ obtained for $Y^{j} . G C$ (resp. $G C^{*}$ ) are the guaranteed costs of $Y$ (resp. in $\mathrm{AC}^{*}$ ). DVals is a list of $n$ arrays 

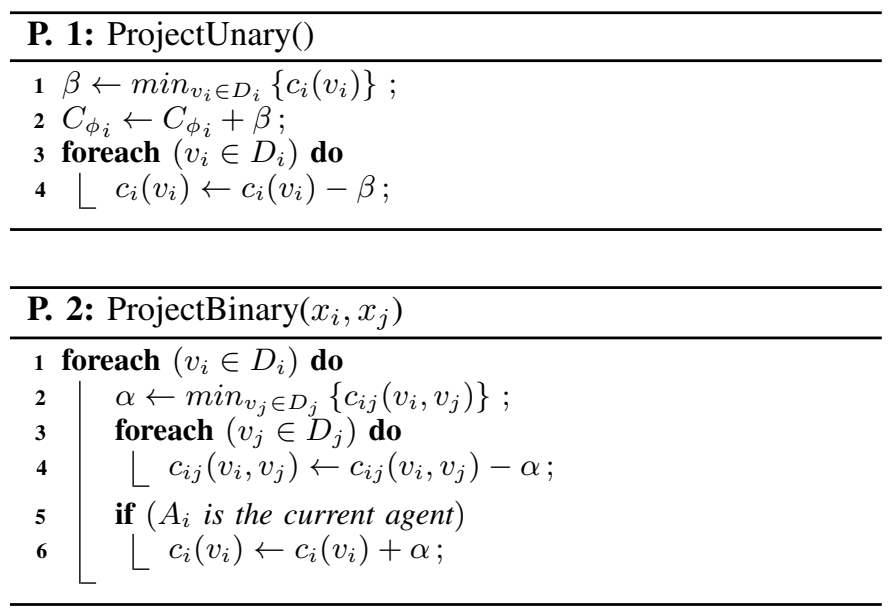

containing deleted values. Each array, DVals $[j]$, contains two elements, listVals which is the list of values deleted by $A_{j}$ and UnvNbrs which is a counter of the $A_{j}$ neighbors that have not yet processed listVals. EVals is a list of arrays containing extension values.

The guaranteed cost of $Y(1)$ is the sum of $c_{i j}$ involved in $Y$.

$$
G C(Y)=\sum_{C_{i j} \in \mathcal{C}} c_{i j}\left(v_{i}, v_{j}\right), \quad\left(x_{i}, v_{i}\right),\left(x_{j}, v_{j}\right) \in Y
$$

If a CPA $Y$ comprises a value assignment for each variable of a given DCOP, then it is called a complete assignment (i.e., a solution). This solution is said to be optimal (2) when the sum of all the constraint costs that it implies is minimal.

$$
Y^{*}=\underset{Y}{\arg \min }\{G C(Y) \mid \operatorname{var}(Y)=\mathcal{X}\}
$$

Fig.1 shows an example of a DCOP in which each agent $A_{i} \in \mathcal{A}=\left\{A_{1}, A_{2}, A_{3}\right\}$ takes control of a single variable $x_{i} \in \mathcal{X}=\left\{x_{1}, x_{2}, x_{3}\right\}$, each being defined on a domain of values $D_{i}=\{0,1\}$ in $\mathcal{D}=\left\{D_{1}, D_{2}, D_{3}\right\}$. Each pair of variables in $\mathcal{X}$ is connected by a binary constraint $C_{i j} \in \mathcal{C}=$ $\left\{C_{12}, C_{13}, C_{23}\right\}$. The costs of the combinations of values of each constraint $C_{i j}$ are indicated in the side tables.

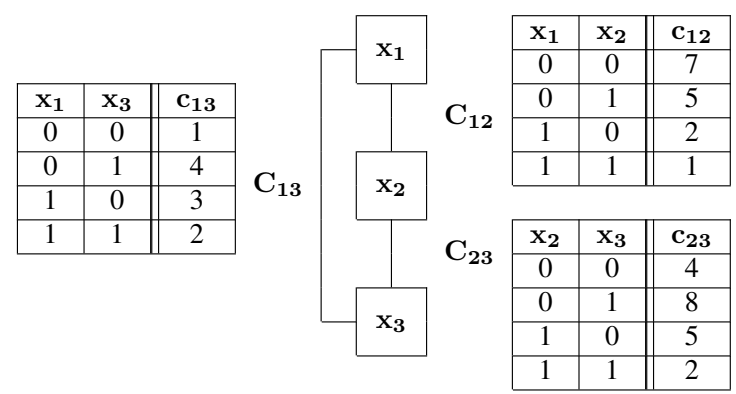

Fig. 1. Example of a distributed constraint optimization problem (DCOP).

\section{B. Soft Arc Consistency Techniques}

Soft arc consistency techniques are used when solving a given problem to delete values that are not part of the optimal solution of that problem. To apply these techniques, we use a set of transformations known as equivalence preserving
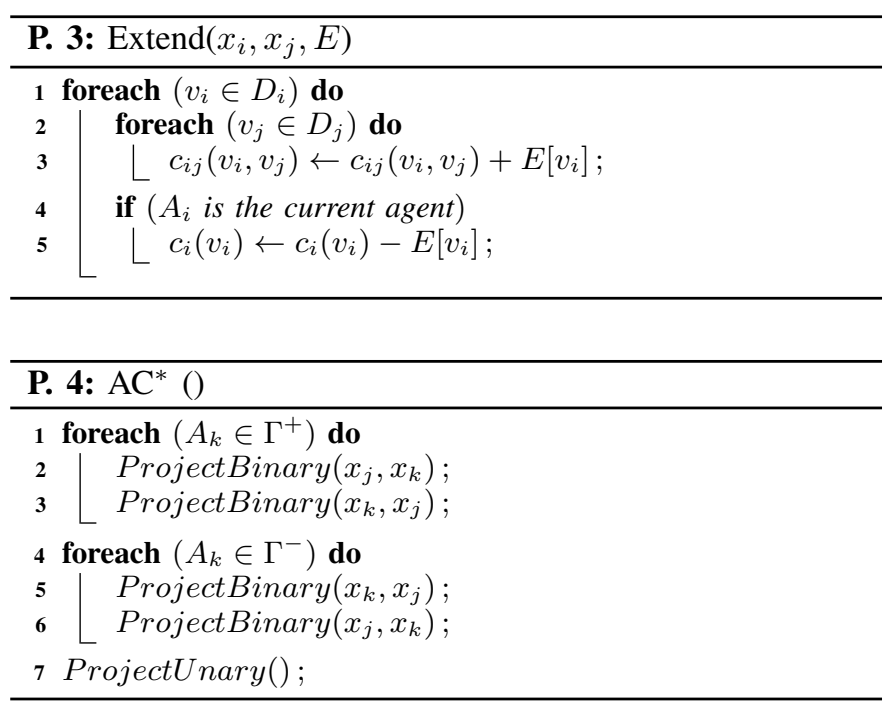

transformations. They allow the exchange of costs between the constraints of the problem according to three manners that are a binary projection, a unary projection, and an extension.

The binary projection (P. 2) is an operation that subtracts, for a value $v_{i}$ of $D_{i}$, the smallest cost $\alpha$ of a binary constraint $C_{i j}$ and adds it to the unary constraint $C_{i}$.

The unary projection (P. 1) is an operation that subtracts the smallest cost $\beta$ of a unary constraint $C_{i}$ and adds it to the zero-arity constraint $C_{\phi}$

The extension (P. 3) is an operation that subtracts, for a value $v_{i}$ of $D_{i}$, the extension value $\left(E\left[v_{i}\right]\right)$ of $v_{i}$ from a unary constraint $C_{i}$ and adds it to the binary constraint $C_{i j}$, with $0<E\left[v_{i}\right] \leq c_{i}\left(v_{i}\right)$

All of these transformations are applied to a problem under a set of conditions represented by soft arc consistency levels [16], namely:

Node Consistency $\left(N C^{*}\right)$ : a variable $x_{i}$ is $\mathrm{NC}^{*}$ if each value $v_{i} \in D_{i}$ satisfies $C_{\phi}+c_{i}\left(v_{i}\right)<U B_{i}$ and there is a value $v_{i} \in D_{i}$ with $c_{i}\left(v_{i}\right)=0$. A problem is $\mathrm{NC}^{*}$ if each variable $x_{i}$ of this problem is $\mathrm{NC}^{*}$.

Arc Consistency $\left(A C^{*}\right)$ : a variable $x_{i}$ is $\mathrm{AC}^{*}$ with respect to its neighbor $x_{j}$ if $x_{i}$ is $\mathrm{NC}^{*}$ and there is, for each value $v_{i} \in D_{i}$, a value $v_{j} \in D_{j}$ which satisfies $c_{i j}\left(v_{i}, v_{j}\right)=0 . v_{j}$ is called a simple support of $v_{i}$. A problem is $\mathrm{AC}^{*}$ if each variable $x_{i}$ of this problem is $\mathrm{AC}^{*}$.

Directional Arc Consistency $\left(D A C^{*}\right)$ : a variable $x_{i}$ is DAC* with respect to its lower neighbor $x_{j(j>i)}$ if $x_{i}$ is $\mathrm{NC}^{*}$ and there is, for each value $v_{i} \in D_{i}$, a value $v_{j} \in D_{j}$ which satisfies $c_{i j}\left(v_{i}, v_{j}\right)+c_{j}\left(v_{j}\right)=0 . v_{j}$ is called a full support of $v_{i}$. A problem is DAC* if each variable $x_{i}$ of this problem is DAC* with its lower neighbors $x_{j(j>i)}$.

Full Directional Arc Consistency $\left(F D A C^{*}\right)$ : A problem is FDAC $^{*}$ if this problem is $\mathrm{AC}^{*}$ and $\mathrm{DAC}^{*}$.

To make any problem $\mathrm{AC}^{*}$, it is necessary to apply, for each variable of this problem, a binary projection (P. 2), then a unary projection (P. 1), and finally a deletion of non-NC* values. These three instructions are repeated each time a value is deleted. In a distributed case, each agent $A_{i}$ performs $\mathrm{AC}^{*}$ locally (P. 4) and shares its contribution value stored in $C_{\phi_{i}}$ (P. 

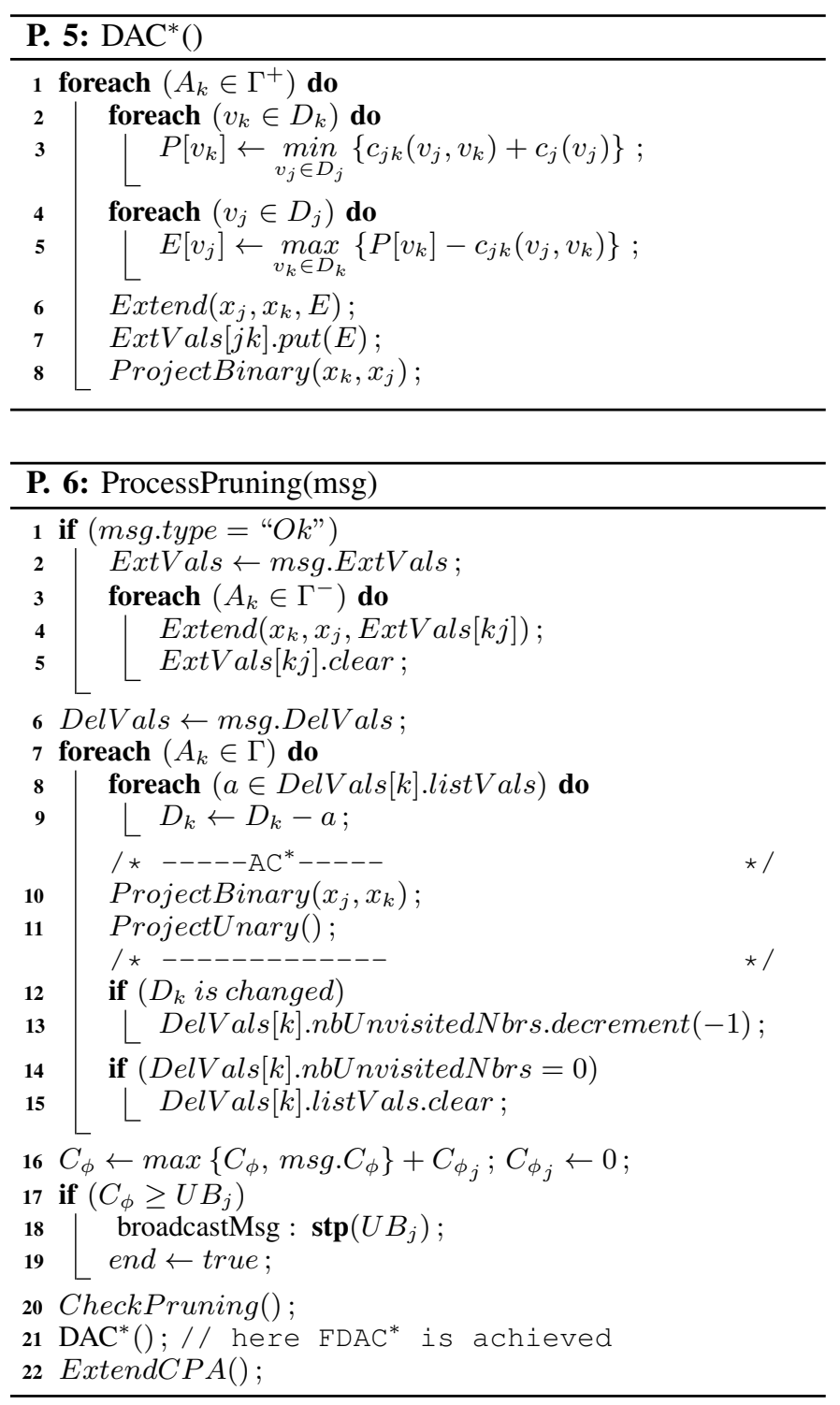

1, line 2) with the other agents in order to calculate the global $C_{\phi}$ (i.e., $C_{\phi}=\sum_{A_{i} \in \mathcal{A}} C_{\phi_{i}}$ ). Each agent $A_{i}$ keeps locally for each of its constraints $C_{i j}$ an identical copy marked by $C_{i j}^{a c}$ and used in $\mathrm{AC}^{*}$ procedure. During $\mathrm{AC}^{*}, C_{i j}^{a c}$ constraints are changed. To keep the symmetry of these constraints in the agents, each agent $A_{i}$ applies, on its copy $C_{i j}^{a c}$, the same action of its neighbor $A_{j}$ and vice versa (P. 4, line 3, 5) [14].

In the same way, we can make any problem DAC*. But in this case, we must first extend (P. 3), for each variable, from its unary costs to its binary costs, the minimum cost required to perform again $\mathrm{AC}^{*}$ by its lower neighbors (P. 5).

By executing $\mathrm{AC}^{*}$ and $\mathrm{DAC}^{*}$ successively for each variable, we can make the problem FDAC**

\section{C. $A F B \_B J^{+} \_A C^{*}$ Algorithm}

Each agent $A_{j}$ carries out the $\mathrm{AFB} \_\mathrm{BJ}^{+}{ }_{-} \mathrm{AC}^{*}$ [3] [2] according to three phases. First, $A_{j}$ initializes its data structures and performs $\mathrm{AC}^{*}$ to delete suboptimal values from its domain $D_{j}$. Second, $A_{j}$ chooses, for its variable $x_{j}$, a value from its previously filtered domain $D_{j}$ in order to extend the CPA

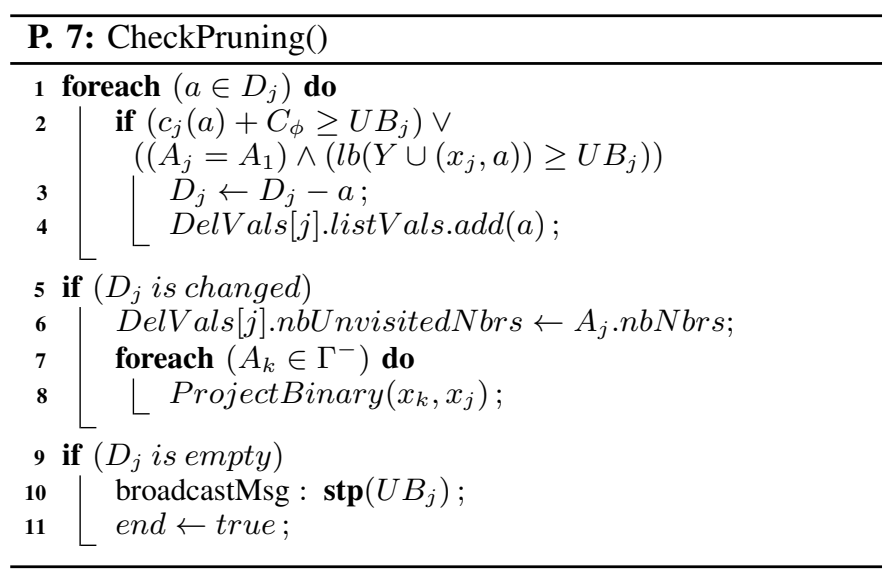

$Y^{j}$ by its value assignment $\left(x_{j}, v_{j}\right)$. If $A_{j}$ has successfully extended the CPA, it sends an ok? message to the next agent asking it to continue the extension of CPA $Y^{j}$. This message loads the extended CPA $Y^{j}$, its guaranteed cost (3), its guaranteed cost of $\mathrm{AC}^{*}(4)$, the $C_{\phi}$, and the list $D V$ als.

$$
\begin{aligned}
& G C\left(Y^{j}\right)[j]=G C\left(Y^{j-1}\right)+\sum_{\left(x_{i}, v_{i}\right) \in Y^{j-1} \mid i<j} c_{i j}\left(v_{i}, v_{j}\right) \\
& \underset{\left(x_{i}, v_{i}\right) \in Y^{j-1}}{G C^{*}\left(Y^{j}\right)}=G C^{*}\left(Y^{j-1}\right)+c_{j}\left(v_{j}\right)+\sum_{C_{i j}^{a c} \in \mathcal{C}} c_{i j}\left(v_{i}, v_{j}\right)
\end{aligned}
$$

In case $A_{j}$ fails to extend the CPA, either because it doesn't find a value that gives a valid CPA, or because all the values in its domain are exhausted, it stops the CPA extension and sends a back message, containing the same data structures as an ok? message excluding $G C$ and $G C^{*}$, to the appropriate agent. If such an agent does not exist or the domain of $A_{j}$ becomes empty, $A_{j}$ stops its execution and informs the others via stp messages. A CPA $Y^{j}$ is said to be valid if its lower bound (5) does not exceed the global upper bound $U B_{j}$, which represents the cost of the optimal solution achieved so far.

$$
L B\left(Y^{j}\right)[i]=G C\left(Y^{j}\right)[i]+\sum_{A_{k}>A_{j}} L B_{k}\left(Y^{j}\right)[i]
$$

Third, $A_{j}$ evaluates the extended CPA by sending fb? messages, which hold the same data structures as an ok? message excluding $C_{\phi}$ and $D V$ als, to unassigned agents asking them to evaluate the CPA and send the result of the evaluation. When an agent has completed its evaluation, it sends the result directly to the sender agent via an lb message. The evaluation is based on the calculation of appropriate lower bounds for the received CPA $Y^{i}$. The lower bound of $Y^{i}(6)$ is the minimum lower bound over all values of $D_{j}$ with respect to $Y^{i}$. 


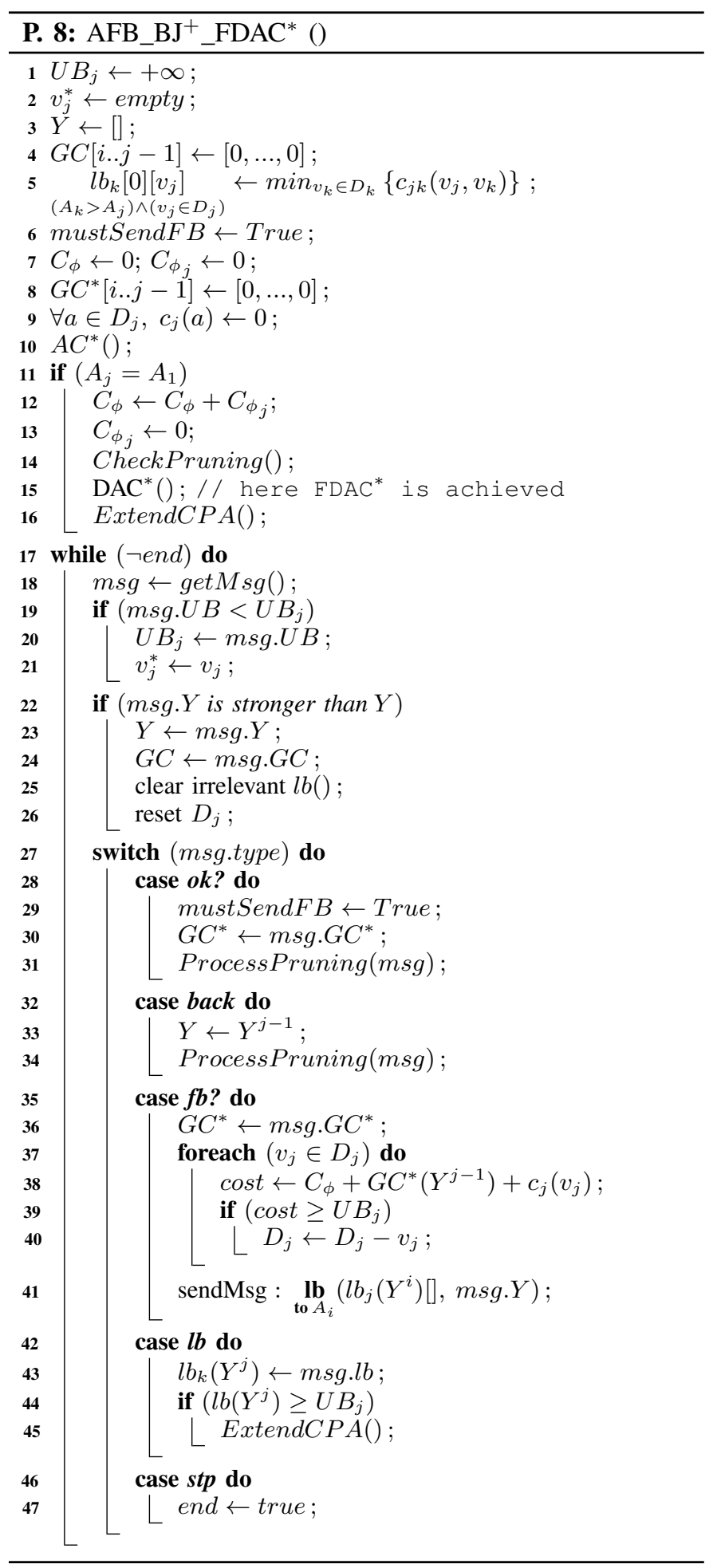

$$
\begin{gathered}
\operatorname{LB}_{j}\left(Y^{i}\right)[h]=\min _{v_{j} \in D_{j}}\left\{\sum_{\substack{\left(x_{k}, v_{k}\right) \in Y^{h} \\
(k \leq h)}} c_{k j}\left(v_{k}, v_{j}\right)+\right. \\
\sum_{\substack{k=h+1 \\
(h<k<i)}}^{i-1} \min _{v_{k} \in D_{k}}\left\{c_{k j}\left(v_{k}, v_{j}\right)\right\}+c_{i j}\left(v_{i}, v_{j}\right)+ \\
\left.\sum_{\substack{x_{k} \in \Gamma^{+}\left(x_{j}\right) \\
(k>j)}} \min _{v_{k} \in D_{k}}\left\{c_{j k}\left(v_{j}, v_{k}\right)\right\}\right\}
\end{gathered}
$$

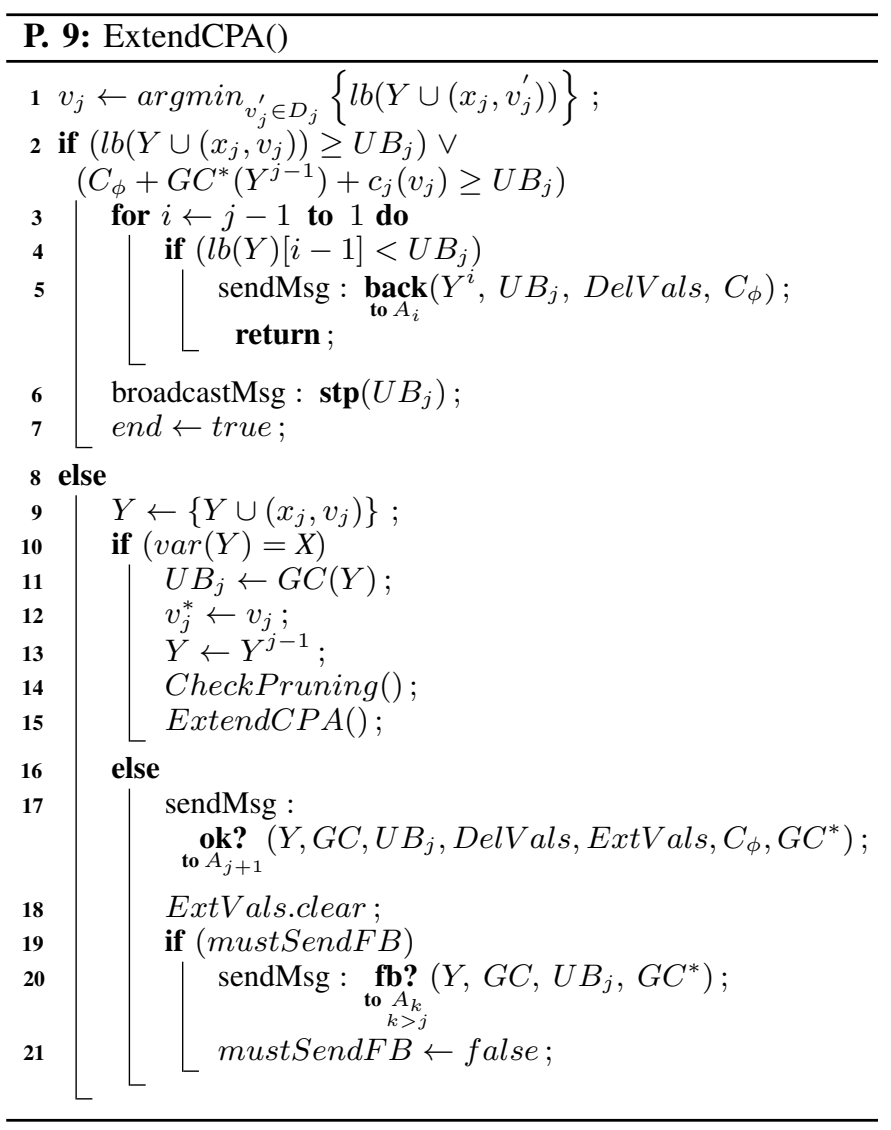

\section{D. $A F B \_B J^{+} \_D A C^{*}$ Algorithm}

The AFB_BJ ${ }^{+}$DAC $^{*}[4]$ algorithm follows the same steps as $\mathrm{AFB} \_\mathrm{BJ}^{+}{ }_{-} \mathrm{AC}^{*}$ algorithm except that it performs $\mathrm{DAC}^{*}$ instead of $\mathrm{AC}^{*}$. With $\mathrm{AC}^{*}$, we can find for each value of a given agent the corresponding simple support in the domains of its lower and higher neighbors. While with $\mathrm{DAC}^{*}$ which is the next level of $\mathrm{AC}^{*}$ and the best in reducing the domains of a given DCOP, we can find for each value of a given agent the corresponding full support in the domains of its lower neighbors only (§II-B).

\section{THE AFB_BJ ${ }^{+}$FDAC $^{*}$ AlgorithM}

In AFB_BJ ${ }^{+}$FDAC* $^{*}$ algorithm, instead of using $\mathrm{AC}^{*}$ and DAC $^{*}$ separately as in previous versions, we use FDAC* which provides the same effect of both together.

FDAC $^{*}$ as mentioned in section II-B is executed by executing $\mathrm{AC}^{*}$ and $\mathrm{DAC}^{*}$ successively. This allows getting for each domain value of each variable simple support in the domains of its higher neighbors $\left(\Gamma^{-}\right)$and full support in the domains of its lower neighbors $\left(\Gamma^{+}\right)$. With FDAC ${ }^{*}$, we can continuously exchange costs between agents, from unary constraints to binary ones and vice versa. This allows the unary costs of each agent and the global zero-arity constraint $\left(C_{\phi}\right)$ to be continuously updated. So, with these updates, we can significantly reduce the agent domain. In short, FDAC ${ }^{*}$ is a technique that allows agents to choose more precisely the best values for their variables by removing more and more invalid values in their domains. 
The skeleton of AFB_BJ ${ }^{+}$FDAC $^{*}$ algorithm is different from those of $\mathrm{AFB} \_\mathrm{BJ}^{+}{ }_{-} \mathrm{AC}^{*}$ and $\mathrm{AFB} \_\mathrm{BJ}^{+}{ }_{-} \mathrm{AC}^{*}$ in two things:

The first one is the $\mathrm{DAC}^{*}$ procedure (P. 5), which is responsible for finding, for each value of an agent, its full support in the domains of its lower neighbors. In DAC* procedure, only a part of the unary costs of a given agent is transferred to its lower neighbors as extension values, not the total of those costs as in AFB_BJ ${ }^{+}{ }_{-} \mathrm{DAC}{ }^{*}$ algorithm. This is so that the $\mathrm{AC}^{*}$ condition that this agent must keep with its higher neighbors is not violated (P. 5, line 2-5) [16].

The second is the condition (P. 7, line 2) that allows the first agent to permanently delete the values having a global lower bound exceeding the global upper bound and the values that it has already evaluated. This condition remains correct only for the first agent according to the static order of agents. This is because the first agent does not have a previous agent, which allows it to permanently delete any value that proved to be inconsistent.

\section{A. Description of $A F B \_B J^{+} \_F D A C^{*}$}

The AFB_BJ ${ }^{+}$FDAC $^{*}$ (P. 8) is performed by each agent $A_{j}$ as follows :

$A_{j}$ starts with the initialization step (P. 8, line 1-10) in which it performs the $\mathrm{AC}^{*}$ (P. 4). If $A_{j}$ is the $1^{\text {st }}$ agent (P. 8, line 11), it filters its domain by calling CheckPruning() (P. 7), then performs $\mathrm{DAC}^{*}()$ (P. 5) after $\mathrm{AC}^{*}$ to ensure the achievement of the FDAC*, and finally calls ExtendCPA() to generate a CPA $Y$.

Next, $A_{j}$ starts processing the messages (P. 8, line 17). First, it updates $U B_{j}$ and $v_{j}^{*}$ (P. 8, line 21). Then, $A_{j}$ updates $Y$ and $G C$ and erases all unrelated lower bounds if the received CPA (msg.Y) is fresh compared to the local one $(Y)$ (P. 8, line 22). Thereafter, $A_{j}$ restores all temporarily deleted values (P. 8, line 40).

When receiving an ok? message (P. 8, line 28), $A_{j}$ authorizes the sending of fb? messages and calls ProcessPruning() (P. 6).

When calling ProcessPruning() (P. 6), $A_{j}$ deals initially, for ok? messages only, with extensions of its higher neighbors (P. 6, line 1-5). Afterward, it updates its DVals, then its neighbors' domains separately in order to keep the same domains as these agents (P. 6, line 6-9). After that, it performs once more the $\mathrm{AC}^{*}$ (P. 6, line 10-11). Next, $A_{j}$ decrements the unvisited neighbors of $A_{k}, D V a l s[k] . U n v N b r s$, and then checks whether it is the last visited neighbor of this agent $A_{k}$ in order to reset its list of deleted values $D$ Vals $[k]$.list Vals (P. 6, line 12-15). Then, $A_{j}$ updates its global $C_{\phi}$ (P. 6, line 16). If $C_{\phi}$ exceeds the $U B_{j}, A_{j}$ turns off its execution and notifies the others (P. 6, line 17-19). Finally, $A_{j}$ calls CheckPruning () to prune its domain, $\operatorname{DAC}^{*}()$ (P. 5) to achieve FDAC*, and ExtendCPA() to extend the received CPA (P. 6, line 20-22).

When calling $\mathrm{DAC}^{*}()$ (P. 5), $A_{j}$ performs the proper extensions from $C_{j}$ to each $C_{i j}$ (P. 5, line 6-7). To do that, $A_{j}$ calculates, for each value $v_{j}$ of $D_{j}$, its extension value (P. 5 , line 4-5) based on the prior computation of the values of the
TABLE I

TOTAL OF MESSAGES ( $m s g s$ ) SENT AND NON-CONCURRENT CONSTRAINT CHECKS (ncccs) FOR SOFT GRAPH COLORING, $p_{1}=0.4$

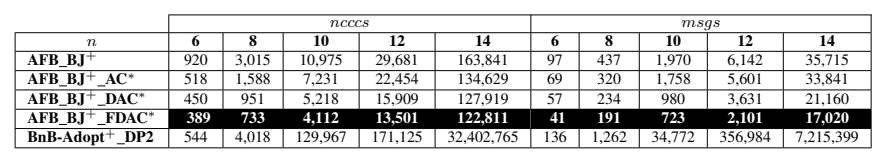

TABLE II

TOTAL OF MESSAGES $(m s g s)$ SENT AND NON-CONCURRENT CONSTRAINT CHECKS (ncccs) FOR SOFT GRAPH COLORING, $p_{1}=0.7$
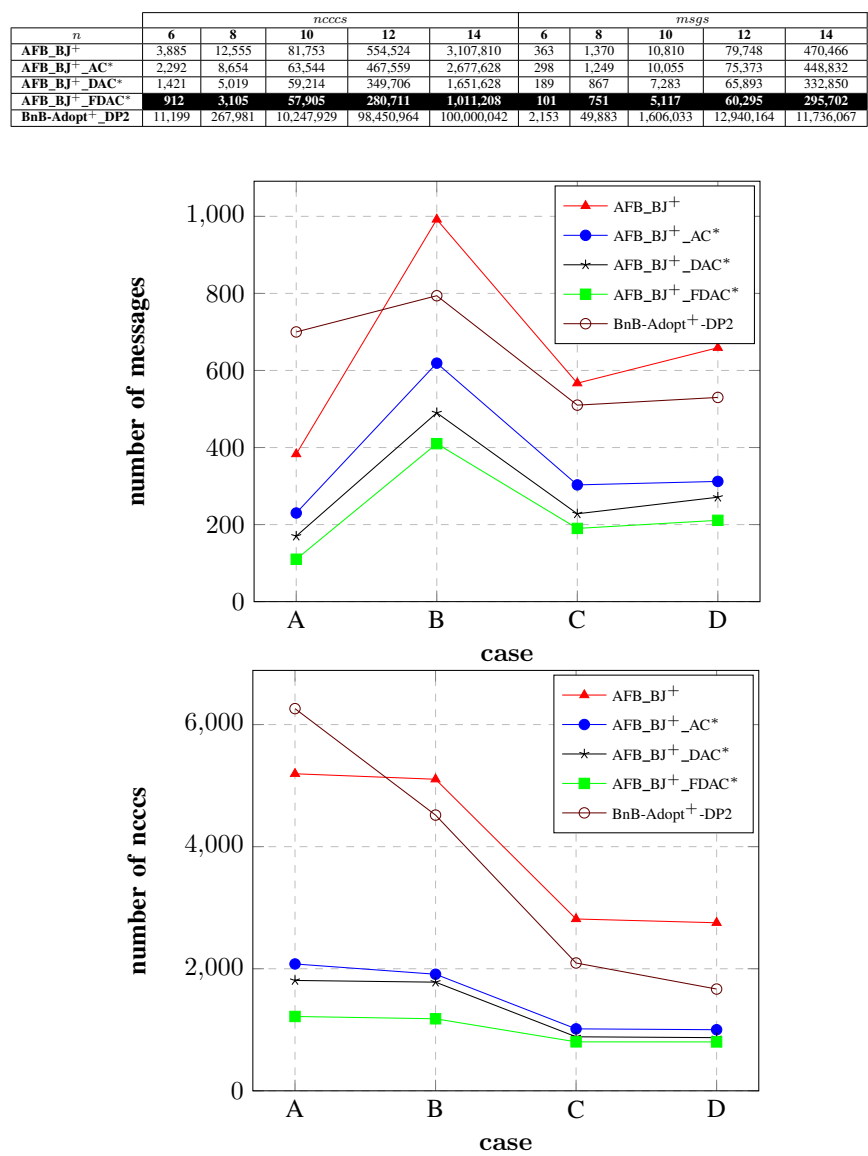

Fig. 2. Total of messages (msgs) sent and non-concurrent constraint checks (ncccs) for meetings scheduling

later projections on its lower neighbors (P. 5, line 2-3) [16]. Once completed, $A_{j}$ performs a binary projection to keep the symmetry of $C_{i j}^{a c}$ constraints (P. 5, line 8). It should be noted that the direction taken into account by each agent $A_{j}$ for the extension of its costs is towards its lower neighbors $\left(\Gamma^{+}\left(x_{j}\right)\right)$.

When calling CheckPruning() (P. 7), $A_{j}$ deletes any value from its domain for which the sum of the $C_{\phi}$ with the unary cost of this value exceeds $U B_{j}$. If $A_{j}$ is the first agent, it also deletes any value whose global lower bound exceeds $U B_{j}$ and any value has already been evaluated (P. 7, line 2-3). With each new deletion, $A_{j}$ initializes the number of its neighbors not yet visited (P. 7, line 5-6). Then, it performs a binary projection to keep the symmetry of $C_{i j}^{a c}$ constraints (P. 7, line 8). If $A_{j}$ domain becomes empty, $A_{j}$ turns off its execution and notifies the others (P. 7, line 9-11). 

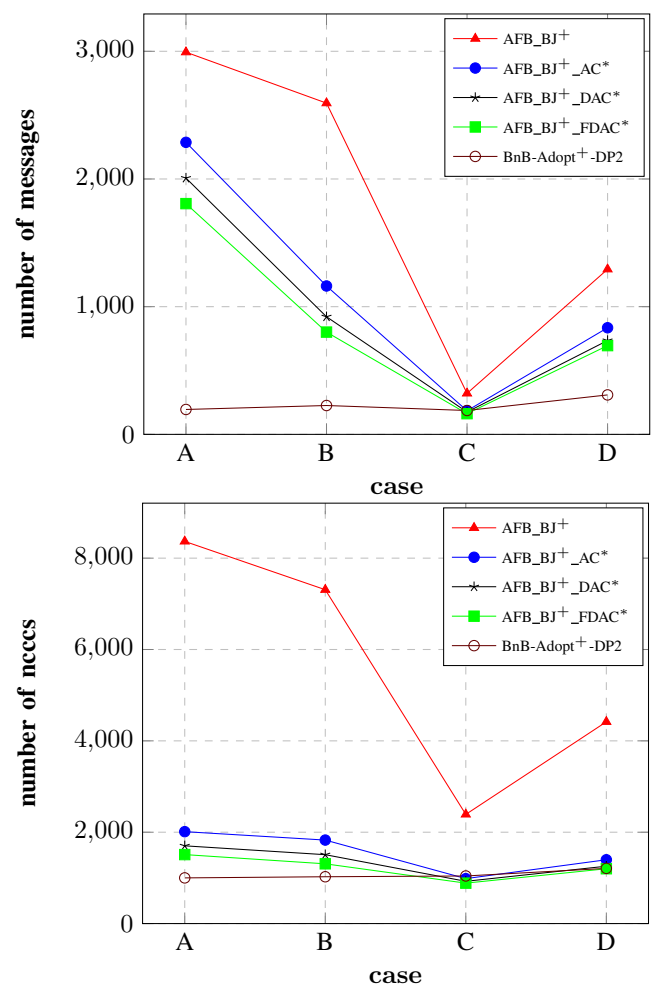

Fig. 3. Total of messages (msgs) sent and non-concurrent constraint checks (ncccs) for sensors network

When calling ExtendCPA() (P. 9), $A_{j}$ looks for a value $v_{j}$ for its variable $x_{j}$ (P. 9 , line 1 ). If no value exists, $A_{j}$ returns to the priority agents by sending a back message to the contradictory agent (P. 9, line 2-5). If no agent exists, $A_{j}$ turns off its execution and notifies the others via stp messages (P. 9 line 6-7). Otherwise, $A_{j}$ extends $Y$ by adding its assignment (P. 9, line 9). If $A_{j}$ is the last agent (P. 9, line 10) then a new solution is obtained and the $U B_{j}$ is updated, which obliges $A_{j}$ to call CheckPruning() to filter again its domain and then ExtendCPA() to proceed the search (P. 9, line 1115). Otherwise, $A_{j}$ sends an ok? message loaded with the extended $Y$ to the next agent (P. 9, line 17) and fb? messages to unassigned agents (P. 9, line 20).

When $A_{j}$ receives an fb? message, it filters its domain $D_{j}$ with respect to the received $Y$ (P. 8, line 36-40), calculates the appropriate lower bounds (6), and immediately sends them to the sender via lb message (P. 8, line 41).

When $A_{j}$ receives an $\mathbf{l b}$ message, it stores the lower bounds received (P. 8, line 43) and performs ExtendCPA() to modify its assignment if the lower bound calculated, based on the cost of $Y(5)$, exceeds the $U B_{j}$.

\section{B. Correctness of $A F B \_B J^{+} \_F D A C^{*}$}

Theorem 1. $A F B \_B J^{+} \_F D A C^{*}$ is guaranteed to calculate the optimum and terminates.

Proof. The AFB_BJ ${ }^{+}$FDAC* ${ }^{*}$ algorithm overrides its previous versions by performing both $\mathrm{AC}^{*}$ and $\mathrm{DAC}^{*}$, which is essentially just a set of cost extensions performed between an agent and its neighbors after performing $\mathrm{AC}^{*}$. These extensions have already been proved which are correct in [16] [8], and they are executed by the AFB_BJ ${ }^{+}$FDAC $^{*}$ without any cost redundancy (P. 3, line 4), (P. 5, line $\overline{8}$ ), and (P. 6, line $1-5)$.

\section{EXPERIMENTAL RESULTS}

In this section, we experimentally compare $\mathrm{AFB}_{\mathrm{B}}{ }^{+}{ }_{\text {FDAC }}{ }^{*}$ algorithm with its previous versions, $\mathrm{AFB}_{\mathrm{B} J}{ }^{+}, \mathrm{AFB} \_\mathrm{BJ}{ }^{+} \mathrm{AC}^{*}$, and $\mathrm{AFB} \_\mathrm{BJ}^{+}{ }_{-} \mathrm{DAC}^{*}$, and with $\mathrm{BnB}-\mathrm{Adopt}^{+}{ }^{+} \mathrm{DP} 2$ algorithm [6], which is its famous competitor. Three benchmarks are used in these experiments: soft graph coloring, meetings scheduling, and sensors network. All experiments were performed on DisChoco 2.0 platform [22], in which agents are simulated by Java threads that communicate only through message passing.

Soft graph coloring [25]: are defined by $\left(n, c, p_{1}\right)$, which are respectively the number of nodes (i.e., variables), the number of possible colors of each node, and the constraint density. The constraints are applied to adjacent nodes. We evaluated two classes of instances $\left(n=6 . .14, c=8, p_{1}=0.4\right)$ and $\left(n=6 . .14, c=8, p_{1}=0.7\right)$. For the constraint costs, they were randomly selected from the set $\{0, \ldots, 100\}$. For each $p_{1}$, we randomly generated an average of 30 instances.

Meetings scheduling [23]: are defined by $(m, p, t s)$, which are respectively the number of meetings (i.e., variables), the number of participants, and the number of time slots for each meeting. Each participant has a private schedule of meetings and each meeting takes place at a particular location and at a fixed time slot. The constraints are applied to meetings that share participants. We have evaluated 4 cases A, B, C, and D, which are different in terms of meetings/participants [17].

Sensors network [7]: are defined by $(t, s, d)$, which are respectively the number of targets (i.e., variables), the number of sensors, and the number of possible combinations of 3 sensors reserved for tracking each target. A sensor can only track one target at most and each combination of 3 sensors must track a target. The constraints are applied to adjacent targets. We have evaluated 4 cases A, B, C, and D, which are different in terms of targets/sensors [17].

To compare the algorithms, we use two metrics which are the total of messages exchanged ( $m s g s$ ) that represents the communication load and the total of non-concurrent constraint checks (ncccs) that represents the computation effort.

In tables I and II, we display respectively the results of experiments carried out on coloring problems of sparse $\left(p_{1}=\right.$ $0.4)$ and dense $\left(p_{1}=0.7\right)$ graphs. The comparison of these results shows an improvement of $\mathrm{AFB} \_\mathrm{BJ}^{+}{ }_{-} \mathrm{FDAC}^{*}$ algorithm reaching 4,000 messages (resp. 5,000 checks) in the sparse case, and reaching 30,000 messages (resp. 600,000 checks) in the dense case. As for BnB-Adopt ${ }^{+}$_DP2 algorithm, it remains largely delayed compared to the other algorithms.

Regarding meetings scheduling problems (Fig. 2), the results show a clear improvement of AFB_BJ ${ }^{+}$FDAC $^{*}$ compared to others, whether for msgs or for ncccs. But with regard to sensors network problems (Fig. 3), BnB-Adopt ${ }^{+}$_DP2 algorithm retains the pioneering role, despite the superiority of $\mathrm{AFB} \_\mathrm{BJ}^{+}{ }^{+} \mathrm{FDAC}^{*}$ algorithm to its previous versions. 
By analyzing the results, we can conclude that the $\mathrm{AFB}_{-} \mathrm{BJ}^{+}{ }_{\text {_FDAC }}^{*}$ is better than its prior versions, because of the existence of Full Directional Arc Consistency (FDAC ${ }^{*}$ ) that allows agents to reapply $\mathrm{AC}^{*}$ multiple times and thus remove more suboptimal values. Regarding the superiority of BnB-Adopt ${ }^{+}$_DP2 over AFB_BJ ${ }^{+}$FDAC $^{*}$ in sensors network problems, this is mainly due to the arrangement of the pseudo-tree used by this algorithm that corresponds to the structure of these problems, as well as the existence of DP2 heuristic that facilitates the proper choice of values.

\section{CONCLUSION}

In this paper, we have introduced the $\mathrm{AFB} \_\mathrm{BJ}^{+} \mathrm{FDAC}^{*}$ algorithm. It relies on Full Directional Arc Consistency $\left(\mathrm{FDAC}^{*}\right)$ to further reduce the agent domains of a given DCOP and thus quickly reach its optimal solution. FDAC* makes it possible to perform more cost extensions from each agent to its neighbors. This allows reapplying over and over again the $\mathrm{AC}^{*}$, which increases the number of deletions carried out by each agent and thus accelerates the process of solving a problem. Experiments on some benchmarks show that the $\mathrm{AFB}_{-} \mathrm{BJ}^{+}{ }_{\text {_FDAC }}$ algorithm behaves better than its previous versions. As future work, we propose to generalize the use of soft arc consistency in its different levels with DCOP algorithms.

\section{REFERENCES}

[1] Rachid Adrdor, Redouane Ezzahir, and Lahcen Koutti. Connecting $\mathrm{AFB}_{-} \mathrm{BJ}^{+}$with soft arc consistency. International Journal of Computing and Optimization, 5 no. 1:9-20, 2018. [Online]. Available: https://doi.org/10.12988/ijco.2018.857.

[2] Rachid Adrdor, Redouane Ezzahir, and Lahcen Koutti. Consistance d'arc souple appliquée aux problèmes dcop. Journées d'Intelligence Artificielle Fondamentale (JIAF), page 63, 2020. [Online]. Available: https://hal.archives-ouvertes.fr/hal-02951644/document.

[3] Rachid Adrdor and Lahcen Koutti. Enhancing AFB_BJ ${ }^{+}$AC $^{*}$ algorithm In 2019 International Conference of Computer Science and Renewable Energies (ICCSRE), pages 1-7. IEEE, July 2019. [Online]. Available: https://doi.org/10.1109/ICCSRE.2019.8807711.

[4] Rachid Adrdor and Lahcen Koutti. Asynchronous forward-bounding algorithm with directional arc consistency. CEUR Workshop Proceedings (CEUR-WS.org), Vol-2970, 2021. [Online]. Available: http://ceurws.org/Vol-2970.

[5] Rachid Adrdor and Lahcen Koutti. Using directional arc consistency with asynchronous forward-bounding algorithm. CEUR Workshop Proceedings (CEUR-WS.org), Vol-2987, 2021. [Online]. Available: http://ceurws.org/Vol-2987.

[6] Syed Ali, Sven Koenig, and Milind Tambe. Preprocessing techniques for accelerating the dcop algorithm adopt. In Proceedings of the fourth international joint conference on Autonomous agents and multiagent systems, pages 1041-1048. ACM, 2005. [Online]. Available: https://dl.acm.org/citation.cfm?id=1082631.

[7] Ramón Béjar, Carmel Domshlak, Cèsar Fernández, Carla Gomes, Bhaskar Krishnamachari, Bart Selman, and Magda Valls. Sensor networks and distributed csp: communication, computation and complexity. Artificial Intelligence, 161(1-2):117-147, 2005. [Online]. Available: https://doi.org/10.1016/j.artint.2004.09.002.

[8] Martin C Cooper, Simon De Givry, Martı Sánchez, Thomas Schiex, Matthias Zytnicki, and Tomáš Werner. Soft arc consistency revisited. Artificial Intelligence, 174(7-8):449-478, 2010. [Online]. Available: https://doi.org/10.1016/j.artint.2010.02.001.

[9] Ferdinando Fioretto, Enrico Pontelli, and William Yeoh. Distributed constraint optimization problems and applications: A survey. arXiv e-prints, pages arXiv-1602, 2016. [Online]. Available: https://arxiv.org/pdf/1602.06347.
[10] Ferdinando Fioretto, Enrico Pontelli, and William Yeoh. Distributed constraint optimization problems and applications: A survey. Journal of Artificial Intelligence Research, 61:623-698, 2018. [Online].Available: https://doi.org/10.1613/jair.5565.

[11] Amir Gershman, Amnon Meisels, and Roie Zivan. Asynchronous forward bounding for distributed cops. Journal of Artificial Intelligence Research, 34:61-88, 2009. [Online]. Available: https://doi.org/10.1613/jair.2591.

[12] Tal Grinshpoun, Tamir Tassa, Vadim Levit, and Roie Zivan. Privacy preserving region optimal algorithms for symmetric and asymmetric dcops. Artificial Intelligence, 266:27-50, 2019. [Online]. Available: https://doi.org/10.1016/j.artint.2018.08.002.

[13] Patricia Gutierrez and Pedro Meseguer. Saving messages in adopt-based algorithms. In Proc. 12th DCR workshop in AAMAS-10, pages 53-64. Citeseer, 2010. [Online]. Available: http://www.iiia.csic.es/files/pdfs/redunbnb-adopt-final.pdf.

[14] Patricia Gutierrez and Pedro Meseguer. Improving bnb-adopt+-ac. In Proceedings of the 11th International Conference on Autonomous Agents and Multiagent Systems-Volume 1, pages 273-280. International Foundation for Autonomous Agents and Multiagent Systems, 2012. [Online] Available: https://dl.acm.org/citation.cfm?id=2343615.

[15] Katsutoshi Hirayama and Makoto Yokoo. Distributed partial constraint satisfaction problem. In International Conference on Principles and Practice of Constraint Programming, pages 222-236. Springer, 1997. [Online]. Available: https://doi.org/10.1007/BFb0017442.

[16] Javier Larrosa and Thomas Schiex. In the quest of the best form of local consistency for weighted csp. In IJCAI, volume 3, pages 239-244, 2003. [Online]. Available: https://dl.acm.org/citation.cfm?id=1630694.

[17] Rajiv T Maheswaran, Milind Tambe, Emma Bowring, Jonathan P Pearce, and Pradeep Varakantham. Taking dcop to the real world: Efficient complete solutions for distributed multi-event scheduling. In Proceedings of the Third International Joint Conference on Autonomous Agents and Multiagent Systems-Volume 1, pages 310-317. IEEE Computer Society, 2004. [Online]. Available: https://ink.library.smu.edu.sg/sis_research/935/.

[18] Toshihiro Matsui and Hiroshi Matsuo. A constraint based formalisation for distributed cooperative sensor resource allocation. International Journal of Intelligent Information and Database Systems, 4(4):307-321, 2010. [Online]. Available: https://doi.org/10.1504/IJIIDS.2010.035578.

[19] Pragnesh Jay Modi, Wei-Min Shen, Milind Tambe, and Makoto Yokoo. Adopt: Asynchronous distributed constraint optimization with quality guarantees. Artificial Intelligence, 161(1-2):149-180, 2005. [Online]. Available: https://doi.org/10.1016/j.artint.2004.09.003.

[20] Duc Thien Nguyen, William Yeoh, Hoong Chuin Lau, and Roie Zivan. Distributed gibbs: A linear-space sampling-based dcop algorithm. Journal of Artificial Intelligence Research, 64:705-748, 2019. [Online]. Available: https://doi.org/10.1613/jair.1.11400.

[21] Alexandra Olteanu, Thomas Léauté, and Boi Faltings. Asynchronous forward bounding (afb): Implementation and performance experiments. Technical report, 2011. [Online]. Available: https://citeseerx.ist.psu.edu/viewdoc/summary?doi=10.1.1.385.4571.

[22] Mohamed Wahbi, Redouane Ezzahir, Christian Bessiere, and ElHoussine Bouyakhf. Dischoco 2: A platform for distributed constraint reasoning. Proceedings of DCR, 11:112-121, 2011. [Online]. Available: http://citeseerx.ist.psu.edu/viewdoc/summary?doi=10.1.1.208.4895.

[23] Mohamed Wahbi, Redouane Ezzahir, and Christian Bessiere. Asynchronous forward bounding revisited. In International Conference on Principles and Practice of Constraint Programming, pages 708-723. Springer, 2013. [Online]. Available: https://doi.org/10.1007/978-3-64240627-0_52.

[24] Mohamed Wahbi, Younes Mechqrane, Christian Bessiere, and Kenneth N Brown. A general framework for reordering agents asynchronously in distributed csp. In International Conference on Principles and Practice of Constraint Programming, pages 463-479. Springer, 2015. [Online]. Available: https://dl.acm.org/doi/abs/10.5555/3102787.3102821.

[25] William Yeoh, Ariel Felner, and Sven Koenig. Bnb-adopt: An asynchronous branch-and-bound dcop algorithm. Journal of Artificial Intelligence Research, 38:85-133, 2010. [Online]. Available: https://doi.org/10.1613/jair.2849. 


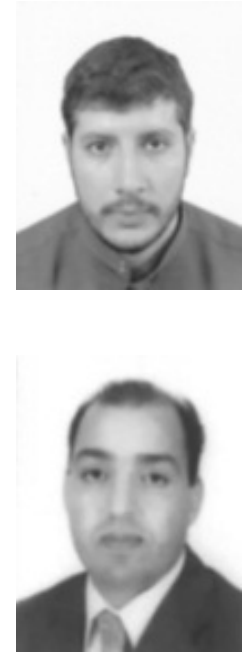

Rachid Adrdor is currently a member of the Laboratory of Computer Information Systems and Vision of the Faculty of Science, Ibn Zohr University, Agadir, Morocco. He received the Ph.D. degree in Computer Science at the Faculty of Science of the same university. His research interests include Applied Artificial Intelligence, in particular, Distributed Constraint Optimization Problems (DCOPs) and Soft Arc Consistency.

Lahcen Koutti is currently a Professor at Department of Computer Science and a member of the Laboratory of Computer Information Systems and Vision of the Faculty of Science, Ibn Zohr University, Agadir, Morocco. He received the Ph.D. degree in Computational Physics from Paul Verlaine University, France and the Habilitation degree from Ibn Zohr University, Morocco. His research interests include Artificial Intelligence and Computer Vision. 